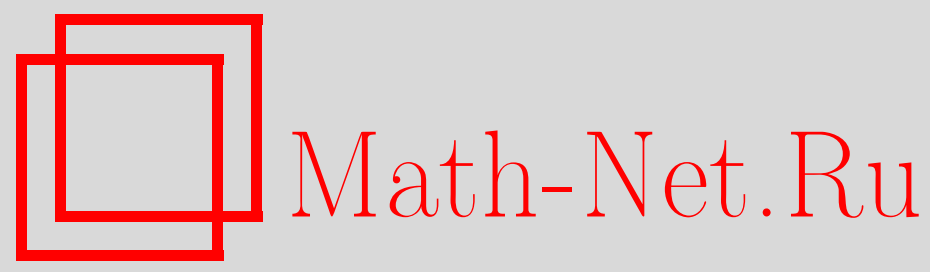

Р. Ш. Оманадзе, О некоторых сводимостях и разбиениях рекурсивно перечислимых множеств, Матем. заметки, 1999, том 66, выпуск 2, 220-230

DOI: https://doi.org/10.4213/mzm1160

Использование Общероссийского математического портала Math-Net.Ru подразумевает, что вы прочитали и согласны с пользовательским соглашением http://www.mathnet.ru/rus/agreement

Параметры загрузки:

IP : 54.198 .64 .247

26 апреля 2023 г., $14: 43: 47$

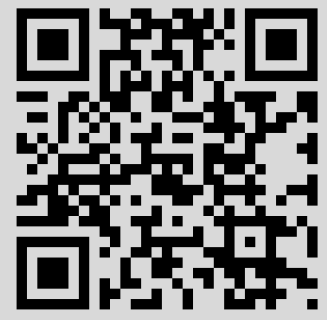




\section{О НЕКОТОРЫХ СВОДИМОСТЯХ И РАЗБИЕНИЯХ РЕКУРСИВНО ПЕРЕЧИСЛИМЫХ МНОЖЕСТВ}

\section{Р.Ш. Оманадзе}

В работе доказано, что существует рекурсивно перечислимая (РП) $T$-степень $a$ такая, что никакое РП полурекурсивное множество $A \in a$ не обладает $Q$-универсальным свойством разбиения. В каждой нерекурсивной РП контигуальной степени существует РП множество $A$ такое, что $A$ имеет универсальное свойство $T$ - $Q$-редукции, но $A$ не является $T$ - $Q$-максимальным множеством. В каждой нерекурсивной РП $W$-степени существует РП множество $A$ такое, что $A$ имеет универсальное свойство $W-s Q$-редукции, но $A$ не является $W$-s $Q$-максимальным множеством. Каждое креативное множество является частично полумаксимальным множеством.

Библиографоия: 21 название.

Рекурсивно перечислимое (РП) разбиение РП множества $A$ - это пара РП множеств $B$ и $C$ такая, что $A=B \cup C$ и $B \cap C=\varnothing$. Если $B$ и $C$ является разбиением множества $A$, то будем писать $A=B \sqcup C$. Пусть $A=B \sqcup C$ и $B, C$ - нерекурсивные РП множества, тогда пара $B$ и $C$ назьвается нетривиальным разбиением множества $A$. Если $A=$ $B \sqcup C$, то $\operatorname{deg}_{T}(A)=\operatorname{deg}_{T}(B) \cup \operatorname{deg}_{T}(C)$. Поэтому РП разбиение является весьма полезным понятием для исследования структуры РП степеней.

РП множество $A$ имеет универсальное свойство разбиения (УСР) [1], [2], если для любого РП множества $D \leqslant{ }_{T} A$ существует разбиение $A=B \sqcup C$ множества $A$ такое, что $B \equiv_{T} D$. Лерман и Реммел $[1],[2]$ показали, что ниже каждой нерекурсивной РП степени существуют как степени, все РП множества которых не обладают УСР, так и степени, содержащие множества с УСР. Кроме того, если $A, B-\mathrm{PП} \mathrm{множества,} A<_{T} B$, то существует РП множество $C$ такое, что $A<_{T} C<_{T} B$ и $C$ не обладает УСР. Последний результат обобщил Довней [3] и показал, что в каждой нерекурсивной РП $T$-степени содержится РП множество, не имеющее УСР.

РП множество $A$ имеет сильное универсальное свойство разбиения [4], если для всех РП множеств $B_{0}, B_{1}$ таких, что $A \equiv_{T} B_{0} \oplus B_{1}$, существует разбиение $A=A_{0} \sqcup A_{1}$ множества $A$ такое, что $A_{0} \equiv_{T} B_{0}$ и $A_{1} \equiv_{T} B_{1}$. Амбос-Спаис и Фейер [4] изучили алгебраические свойства $T$-степеней РП множеств, обладающих (сильно) универсальным свойством разбиения, и получили ряд интересных результатов.

В работе [5] были введены интересные понятия полумаксимального и частично полумаксимального множеств. Довней и Стоб [5] доказали, что если $A, B$ - полумаксимальные множества, то существует $\Phi \in \operatorname{Aut}(\mathscr{E})$ такой, что $\Phi(A)=B$, а если $A$ - час- 
тично полумаксимальное множество, то существуют $B \equiv_{T} \varnothing^{\prime}$ и $\Phi \in \operatorname{Aut}(\mathscr{E})$ такие, что $\Phi(A)=B$, где $\operatorname{Aut}(\mathscr{E})$ есть групша автоморфизмов решетки РП множеств $\mathscr{E}$.

В данной работе доказьвается, что существует РП $T$-степень $a$ такая, что никакое РП полурекурсивноемножество $A \in a$ необладает $Q$-универсальным свойством разбиения. В каждой нерекурсивной РП контигуальной степени существует РП множество $A$ такое, что $A$ имеет универсальное свойство $T$ - $Q$-редукции, но $A$ не является $T$ - $Q$-максимальным множеством. В каждой $\neq 0 \mathrm{P} \Pi W$-степени существует $\mathrm{P} \Pi$ множество $A$ такое, что $A$ имеет универсальное свойство $W$-s $Q$-редукции, но $A$ не является $W$-s $Q$-максимальным множеством. Исследуется разбиение креативного множества и показано, что каждое креативное множество является частично полумаксимальным множеством, что является ответом на вопрос из работы [5]. Все множества, рассматриваемые в данной работе, являются РП множествами.

Все понятия и обозначения, используемые и не определяемые в этой работе, можно найти в [6], [7].

Пусть $r$ - некоторая сводимость.

ОПРЕДЕЛЕНИЕ. РП множество $A$ имеет $r$-универсальное свойство разбиения $(r$-УСР), если для каждого РП множества $B \leqslant r A$ существует РП разбиение $A=A_{0} \sqcup A_{1}$ множества $A$ такое, что $A_{0} \equiv r B$.

Пусть $\varphi_{0}^{B}, \varphi_{1}^{B}, \ldots$ - нумерация функций, рекурсивных относительно $B ; C_{A}$ - характеристическая функция множества $A ;\left\{D_{z}\right\}$ - каноническая нумерация семейства всех конечных подмножеств множества $\omega=\{0,1,2, \ldots\}$.

Говорят, что $A \leqslant{ }_{W} B$, если существует $z$ такое, что $C_{A}(x)=\varphi_{z}^{B}(x)$, и все те числа, вопросы о принадлежности или непринадлежности которых множеству $B$ использовались при вычислении $\varphi_{z}^{B}(x)$, содержатся в $D_{f(x)}$, где $f$ - общерекурсивная функция $(\mathrm{OP} \Phi)$.

Говорят, что $A \leqslant Q \quad B$, если существует ОР $\Phi f$ такая, что

$$
(\forall x)\left(x \in A \Longleftrightarrow W_{f(x)} \subseteq B\right)
$$

Если, кроме того, существует ОР $\Phi$ такая, что

$$
(\forall x)(\forall y)\left(y \in W_{f(x)} \Longrightarrow y<g(x)\right),
$$

то $A \leqslant s Q B$.

Если $A \leqslant s Q B$ и существует фиксированное число $n \in \omega$ такое, что

$$
(\forall x)\left(\left|W_{f(x)}\right|<n\right)
$$

то $A \leqslant{ }_{b s} Q B$.

В работах [1] и [4] были введены понятия: универсальное свойство $W$-редукции и $W$-максимальное множество. Аналогичные понятия естественным образом определяются и для других сводимостей.

Говорят, что $R$-сводимость слабее $r$-сводимости (а $r$ сильнее $R$ ), если $A \leqslant r B \Longrightarrow$ $A \leqslant \leqslant_{R} B$ для всех $A, B \subseteq \omega$.

Пусть $R$-сводимость слабее $r$-сводимости. 
ОПРЕДЕЛЕНИЕ. РП множество $A$ назовем $R-r$-максимальным, если

$$
(\forall B \mathrm{P} \Pi)\left(B \leqslant_{R} A \Longrightarrow B \leqslant_{r} A\right) .
$$

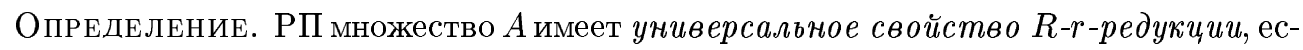
ли

$$
(\forall B \mathrm{P} \Pi)\left(\exists A_{0} \mathrm{P} \Pi\right)\left(B \leqslant_{R} A \Longrightarrow A_{0} \equiv_{r} B \& A_{0} \leqslant r A\right) .
$$

Из теоремы 3.5 и следствия 2.10 работы [8] получается, что если РП $T$-степень $c$ такая, что $c^{\prime}=0^{\prime}$, то существуют РП $T$-степень $a \geqslant c$ и РП множество $A \in a$ такие, что для каждого РП множества $B \leqslant T$ с бесконечньм дополнением существует разбиение $A=A_{0} \sqcup A_{1}$ такое, что $A_{0} \equiv_{m} B$. Таким образом, существует РП множество, обладающее $r$-универсальньм свойством разбиения, где $r$ - произвольная сводимость, промежуточная между $m$ - и $T$-сводимостями.

ПреДЛОЖЕНИЕ 1 (см. [9]). Пусть $A=B \sqcup C$. Тољда $B \leqslant b s Q A u C \leqslant b s Q A$.

СледСтвИЕ. 1) Если РП множество А обладает T-УСР, то $A$ имеет универсальное свойство $T-Q-(T-s Q-, T-b s Q-)$ редукиии.

2) Если РП мнохество А обладает $Q$-УСР, то А имеет универсальное свойст-

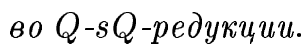

3) Если РП мнохество $A$ обладает $W$-УСР, то $A$ имеет универсальное свойство $W-s Q-$ редукиии.

Множество $A$ называется полурекурсивным [10], если существует двухместная ОР $\Phi$ $g$ такая, что

$$
(\forall x)(\forall y)(g(x, y) \in\{x, y\} \quad \& \quad(\{x, y\} \cap A \neq \varnothing \Longrightarrow g(x, y) \in A))
$$

Лемма 1 (см. [11]). Пусть $B$ - полурекурсивное РП мнохсество, $B \neq \omega, B \neq \varnothing$, $A$ - РП множество и $A$ сводится по Тьюрингу к B. Тогда $A Q$-сводится $\kappa B$.

Лемма 2 (см. [12]). Пусть $B$ - полурекурсивное $\mathrm{P} \Pi$ множество, $B \neq \omega, B \neq \varnothing$, $A$ - РП множество и $A W$-сводится $\kappa$ B. Тогда $A$ sQ-сводится $\kappa B$.

РП T-степень назьвается контигуальной, если в ней все РП множества $W$-эквивалентны.

ЗАмечАниЕ. Существует РП нерекурсивное множество, которое является одновременно $T$ - $Q$-максимальным, $T$-s $Q$-максимальньм, $Q$-s $Q$-максимальным и $W$-s $Q$-максимальным.

Действительно, пусть $a$ - контигуальная РП $T$-степень, $A$ - РП полурекурсивное множество и $A \in a[10]$. Тогда в силу леммы $2 A$-искомое множество.

Теорема 1. Пусть а - РП Т-степень такая, что существует РП T-степень $b \leqslant a$ u $b^{\prime}=0^{\prime \prime}$. Тогда никакое $\mathrm{P} \Pi$ полурекурсивное множсество $A \in$ а не обладает $Q$-универсальным свойством разбиения. 
ДокАЗАТЕЛЬСтвО. Пусть РП $T$-степень $a$ удовлетворяет условию теоремы, $A$ - РП полурекурсивное множество и $A \in a[10]$. Допустим, что $A$ обладает $Q$-универсальным свойством разбиения. Так как по условию теоремы существует РП $T$-степень $b \leqslant a$ и $b^{\prime}=0^{\prime \prime}$, то согласно хорошо известному результату Мартина существует максимальное множество $M$ такое, что $M \leqslant_{T} A$. Тогда в силу леммы $M \leqslant Q A$. Поэтому существует разбиение $A=A_{0} \sqcup A_{1}$ такое, что $M \equiv_{Q} A_{0}$. Тогда в силу теоремы 1 [13] $M \leqslant_{m} A_{0}$. Согласно предложению $1 A_{0} \leqslant b s Q A$, поэтому $M \leqslant b s Q A$. Но в [14] доказано, что если $M$ - максимальное множество и $A-\mathrm{P} П$ полурекурсивное множество, то $\left.M\right|_{b s Q} A$. Следовательно, $M \Varangle_{b s} Q A$. Полученное противоречие доказывает теорему.

Пусть $M$-максимальное множество, $A$ - РП полурекурсивноемножество и $M \leqslant W A$. Тогда в силу леммы $2 M \leqslant_{s Q} A$. Поэтому из теоремы 1 вытекает

СлЕДСтвиЕ. Существует РП полурекурсивное множество, которое не обладает $s Q$-универсальным свойством разбиения.

РП множество $A$ называется нигде не просты. $[15]$, если для каждого РП множества $B$ из того, что $B \backslash A$ бесконечно, следует, что существует бесконечное РП множество $W \subseteq B \backslash A$.

Теорема 2. В каждой нерекурсивной РП контигуальной степени существует РП множество A, обладающее универсальнымм свойством T-Q-редукиии и не являющееся $T$-Q-максимальным.

ДокАЗАТЕЛЬСтво. Известно [15], что в каждой РП $T$-степени существует нигде не простое множество. Пусть $a$ - нерекурсивная РП контигуальная степень, $A$ - нигде не простое множество, $A \in a$ и $A$ является цилиндром. Если $A$ не является цилиндром, то будем рассматривать множество $A \times \omega$ (так как $A \equiv_{m} A \times \omega$, то $A \times \omega$ будет нигде не простым множеством). Тогда в силу следствия 1.12 [4] $A$ имеет сильное универсальное свойство разбиения, т.е. для всех РП множеств $B_{0}, B_{1}$ таких, что $A \equiv_{T} B_{0} \oplus B_{1}$, сушествует разбиение $A=A_{0} \sqcup A_{1}$ такое, что $A_{0} \equiv_{T} B_{0}$ и $A_{1} \equiv_{T} B_{1}$. Пусть $b<a$, $B$ - простое множество и $B \in b$. Тогда существует разбиение $A=A_{0} \sqcup A_{1}$ такое, что $B \equiv_{T} A_{0}$. В силу предложения $1 A_{0} \leqslant Q A$. Известно [16], что если $A$-нигде не простое множество, $C-\mathrm{P} \Pi$ множество и $C \leqslant Q A$, то $C$ - нигде не простое множество. Поэтому $B \nless_{Q} A$.

СлЕДСтвиЕ. В каждой нерекурсивной РП контигуальной T-степени существует РП множество А, обладающее универсальным свойством T-sQ-редукиии и не являющееся $T$-sQ-максимальным.

Tеорема 3. В каждой нерекурсивной РП $W$-степени существует $\mathrm{P} \Pi$ множество $A$, обладающее универсальным свойством $W$-sQ-редукиии и не являющееся $W$-sQ-максимальным множсеством.

ДоказАтельство. Пусть $a$ - нерекурсивная РП $W$-степень, $A \in a$ и $A$ - нигде не простое множество [15]. Допустим, что $A$ является цилиндром. В силу следствия 1.8 [4] 
$A$ имеет $W$-сильное универсальное свойство разбиения, т.е.

$$
\begin{aligned}
& \left(\forall B_{0}\right)\left(\forall B_{1}\right)\left(A \equiv_{W} B_{0} \oplus B_{1}\right. \\
& \left.\quad \Longrightarrow\left(\exists A_{0}\right)\left(\exists A_{1}\right)\left(A=A_{0} \sqcup A_{1} \quad \& \quad A_{0} \equiv_{W} B_{0} \quad \& \quad A_{1} \equiv_{W} B_{1}\right)\right) .
\end{aligned}
$$

Известно [17], что в каждой нерекурсивной РП $W$-степени содержится простое множество. Пусть $b<a, B$ - простое множество и $B \in b$. Тогда существует разбиение $A=A_{0} \sqcup A_{1}$ такое, что $B \equiv_{W} A_{0}$. В силу предложения $1 A_{0} \leqslant_{s Q} A$. Но $B \$_{s} A$, поскольку $B$ - простое множество, а $A$ - нигде не простое множество.

Автору неизвестно, существует ли РП множество, обладающее универсальным свойством $Q$-s $Q$-редукции и не являющееся $Q$-s $Q$-максимальным.

Множество $A b d$-сводится к множеству $B$, если существуют ОР $\Phi f$ и число $n \in \omega$ такие, что

$$
(\forall x)\left(\left(x \in A \Longleftrightarrow D_{f(x)} \cap B \neq \varnothing\right) \&\left|D_{f(x)}\right|<n\right) .
$$

Дичев [18] доказал, что существует РП $b d$-степень, в которой содержится бесконечное число попарно $c$-несравнимых РП множеств. Очевидно, что из $c$-сводимости следует $s Q$-сводимость, обратное неверно. Поэтому приводимое ниже предложение является более обшим утверждением, чем теорема 2 из работы [18].

РП множество $A$ назьвается неускоряемы.м [19], если существуют число $i \in \omega$ и ОР $\Phi$ $h$ такие, что $W_{i}=A$ и

$$
(\forall j)\left(W_{j}=A \Longrightarrow(\forall \stackrel{\infty}{x})\left(x \in A \Longrightarrow \Phi_{i}(x) \leqslant h\left(x, \Phi_{j}(x)\right)\right)\right)
$$

где $\Phi_{u}$ - частично рекурсивная функция, удовлетворяюшая аксиомам Блюма [19], а символом $\forall \stackrel{\infty}{x}$ обозначается выражение “для почти всех $x$ ”.

Если РП множество $A$ не является неускоряемым, то $A$ назьвается ускоряемым множеством.

ПРЕДЛОЖЕНИЕ 2. Пусть РП множество А обладает свойством

$$
\left(\forall A_{0}\right)\left(\forall A_{1}\right)\left(A=A_{0} \sqcup A_{1} \Longrightarrow A_{0} \leqslant b d A \& A_{1} \leqslant b d A\right) .
$$

Тогда если А ускоряемое или $A$ не является нигде не простым множсеством, то в bd-степени множества $A$ содержится бесконечное число попарно sQ-несравнимых РП множеств.

ДокАЗАТЕЛЬСтво. Пусть ускоряемое множество $A$ удовлетворяет условию предложения, $A=A_{0} \sqcup A_{1}, A_{0}$ и $A_{1}$ - неускоряемые множества [7]. Известно [12], что если $A_{0}, A_{1}$ - неускоряемые множества, то $A_{0} \oplus A_{1}$ - неускоряемое множество. Поэтому

$$
A \equiv_{b d} A_{0} \oplus A_{1} \quad \& \quad A_{0} \oplus A_{1}<_{s Q} A .
$$


Тогда в силу теоремы 1 [12] существует бесконечное число попарно $s Q$-несравнимых РП множеств $\left\{C_{i}\right\}_{i \in \omega}$ таких, что

$$
(\forall i)\left(A_{0} \oplus A_{1}<_{m} C_{i}<_{s Q} A\right) .
$$

Из доказательства теоремы 1 [12] следует, что если $A$ удовлетворяет условию предложения, то $(\forall i)\left(C_{i} \equiv_{b d} A\right)$.

Пусть теперь $A$ не является нигде не простым множеством и удовлетворяет условию предложения, $A=A_{0} \sqcup A_{1}, A_{0}$ и $A_{1}$ - нигде не простые множества [15]. Тогда нетрудно проверить, что $A_{0} \oplus A_{1}$ есть нигде не простое множество. В силу предложения 1 [16], если $C$ - РП множество, $B$ - нигде не простое множество и $C \leqslant Q \quad B$, то $C$ - нигде не простое множество. Поэтому

$$
A \equiv_{b d} A_{0} \oplus A_{1} \quad \& \quad A_{0} \oplus A_{1}<_{s Q} A,
$$

и доказательство завершается точно так же, как и в предыдущем случае.

ЗАмЕчАниЕ. Очевидно, что условию предложения 2 удовлетворяют креативные множества, так как известно [15], что креативное множество не является нигде не простым множеством и креативное множество есть ускоряемое множество [19]. Довней и Джокуш [8] доказали, что существует РП T-степень, отличная от 0 и $0^{\prime}$, такая, что в этой $T$-степени имеется наибольшая 1 -степень [8, теорема 1$]$. Ясно, что множество, построенное при доказательстве указанной теоремы 1 [8], удовлетворяет условию предложения 2, так как это множество не может быть нигде не простым.

РП множество $A$ называется полумаксимальным [5], если существует максимальное множество $M$ и нетривиальное разбиение $M_{0}, M_{1}$ множества $M$ такое, что $A=M_{0}$.

РП множество $A$ назьвается частично полумаксимальным [5], если существует нетривиальное разбиение $A_{0}, A_{1}$ множества $A$ такое, что $A_{0}$ является полумаксимальньп.

ПРЕДЛОЖЕНИЕ 3. Каждое креативное множество является частично полумаксимальным множеством.

ДокАЗАТЕЛЬСТво. Известно, что всякое бесконечное РП множество является объединением не пересекающихся между собой креативного и продуктивного множеств. Пусть $M$ - максимальноемножество, $M_{0}, M_{1}$ - нетривиальное разбиениемножества $M$. Допустим, что $M_{1}=A \cup B \& A \cap B=\varnothing$, где $A$ - креативное множество и $B$ - продуктивное множество. Тогда $M_{0} \cup A$ - креативное множество.

Хорошо известно, что все креативные множества рекурсивно изоморфны. Пусть $K-$ креативное множество, $f$ - ОР $\Phi$, взаимно однозначно отображающая множество $\omega$ на себя и $f\left(M_{0} \cup A\right)=K$. Тогда

$$
K=f\left(M_{0}\right) \cup f(A) \& f\left(M_{0}\right) \cap f(A)=\varnothing
$$

и $f\left(M_{0}\right), f(A)$ - РП нерекурсивные множества, т.е. $f\left(M_{0}\right), f(A)$ является нетривиальным разбиением множества $K$. 
Рассмотрим множество $f(M)$. Ясно, что $f(M)$ - максимальное множество и $f\left(M_{0}\right)$, $f\left(M_{1}\right)$ является нетривиальным разбиением максимального множества $f(M)$. Итак, $f\left(M_{0}\right), f(A)$ является нетривиальным разбиением множества $K$, а $f\left(M_{0}\right), f\left(M_{1}\right)$ является нетривиальным разбиением максимального множества $f(M)$.

Если $A \leqslant Q$ $B$ посредством ОР $\Phi f$ и

$$
(\forall x)(\forall y)\left(x \neq y \Longrightarrow W_{f(x)} \cap W_{f(y)}=\varnothing\right)
$$

то множество $A Q_{1}$-сводится к множеству $B\left(A \leqslant Q_{1} B\right)$.

ПРЕДЛОЖЕНИЕ 4. Пусть $H$ - гипер-гиперпростое множество, $B_{0}, B_{1}-$ его нетривиальное разбиение, $K$ - креативное множсество, $A_{0}, A_{1}$ - нетривиальное разбиение $K$ и при этом $A_{1}$ не является креативным множеством. Тогда $A_{0} \nless Q_{1}$ $B_{0}\left(A_{0} \nless_{Q_{1}} B_{1}\right)$.

ДОКАЗАТЕЛЬСТво. Пусть $g-$ ОР $\Phi$ такая, что

$$
(\forall x)\left(x \in A_{0} \Longleftrightarrow W_{g(x)} \subseteq B_{0}\right), \quad(\forall x)(\forall y)\left(x \neq y \Longrightarrow W_{g(x)} \cap W_{g(y)}=\varnothing\right) .
$$

Рассмотрим множество $E=\left\{x: W_{g(x)} \cap B_{1} \neq \varnothing\right\}$. Тогда $A_{0} \cap E=\varnothing$. Множество $A_{0} \cup E$ гипер-гиперпростое. Действительно, если $h-\mathrm{OP} \Phi$ такая, что

$$
(\forall x)(\forall y)\left(x \neq y \Longrightarrow W_{h(x)} \cap W_{h(y)}=\varnothing\right), \quad(\forall x)\left(W_{h(x)} \cap \overline{A_{0} \cup E} \neq \varnothing\right),
$$

то ОР $\Phi \mu$, определенная равенством

$$
(\forall x)\left(W_{\mu(x)}=\bigcup_{i \in W_{h(x)}} W_{g(i)}\right)
$$

противоречит гипер-гиперпростоте множества $H$.

Известно (Деккер), что пересечение креативного и простого множеств является креативным множеством. Поэтому множество $A_{0} \cup\left(E \cap A_{1}\right)$ креативно. Покажем, что $E \cap A_{1}$ не является креативным множеством. Действительно, $E$ не является креативньм множеством, так как $A_{0} \cap E=\varnothing$ и $A_{0} \cup E$ - гипер-гиперпростое множество. По условию $A_{1}$ не является креативным множеством и, кроме того,

$$
(\forall x)\left(x \in E \cap A_{1} \Longleftrightarrow\langle x, x\rangle \in E \times A_{1}\right) .
$$

Тогда если $E \cap A_{1}$ - креативное множество, то в силу теоремы Лахлана [20] $E$ или $A_{1}$ креативно. Противоречие.

Пусть $f$ - одно-однозначная ОР $\Phi$ и $f(\omega)=A_{0} \cup E$. Множество $f^{-1}\left(A_{0} \cup\left(E \cap A_{1}\right)\right)$ креативно. Но $f^{-1}\left(A_{0}\right)$ - рекурсивное множество. Поэтому $f^{-1}\left(E \cap A_{1}\right)$ креативно и, следовательно, $f f^{-1}\left(E \cap A_{1}\right)=E \cap A_{1}$ креативно. Противоречие. 
ЗАмечание. Если $A \leqslant_{c} B$ посредством ОР $\Phi f$ и

$$
(\forall x)(\forall y)\left(x \neq y \Longrightarrow D_{f(x)} \cap D_{f(y)}=\varnothing\right),
$$

то множество $A c_{1}$-сводится к множеству $B$. Аналогично предложению 4 доказьвается, что если $B$ - гиперпростое множество, $B_{0}, B_{1}$ - его нетривиальное разбиение, $K-$ креативное множество, $A_{0}, A_{1}$ - нетривиальное разбиение $K$ и при этом $A_{1}$ не является креативным множеством, то $A_{0} \Varangle_{c_{1}} B_{0}\left(A_{0} \Varangle_{c_{1}} B_{1}\right)$.

Множество $A$ p-сводится к множеству $B[10]$, если существует $\operatorname{OP} \Phi f$ такая, что

$$
(\forall x)\left(x \in A \Longleftrightarrow(\exists y)\left(y \in D_{f(x)} \& D_{y} \subset B\right)\right) .
$$

ПРЕДЛОЖЕнИЕ 5. Пусть $K$ - креативное множество, $A, B$ - нетривиальное разбиение множества $K$ и при этом $B$ не является креативным множеством. Тогда множество $А$ не может р-сводиться $\kappa$ гиперпростому множеству.

ДокАЗАТЕЛЬСТво. Пусть $H$-гиперпростое множество, $K$-креативноемножество, $A, B$ удовлетворяют условиям предложения и

$$
(\exists f \mathrm{OP} \Phi)(\forall x)\left(x \in A \Longleftrightarrow(\exists y)\left(y \in D_{f(x)} \& D_{y} \subset H\right)\right) .
$$

Рассмотрим множество

$$
C=\bigcup_{x \in B} \bigcup_{y \in D_{f(x)}} D_{y}
$$

Возможны два случая:

1) $|C \backslash H|<\infty$;

2) $|C \backslash H|=\infty$.

Рассмотрим эти случаи.

1) Допустим, что $|C \backslash H|<\infty$ и $R=C \backslash H$. Тогда множество

$$
R_{1}=\left\{x:(\forall y)\left(y \in D_{f(x)} \Longrightarrow D_{y} \cap R \neq \varnothing\right)\right\}
$$

рекурсивное и, очевидно, $B \subseteq R_{1} \& R_{1} \cap A=\varnothing$.

Кобзев [21] показал, что если $P$ - продуктивное множество, $A$ - РП множество, то $P \cap A$ или $P \cap \bar{A}$ продуктивно. Поэтому $R_{1} \cap \bar{K}$ или $\bar{R}_{1} \cap \bar{K}$ продуктивно.

Пусть $R_{1} \cap \bar{K}$ продуктивно. Тогда $\bar{R}_{1} \cup B$ креативно и в силу рекурсивности множества $\bar{R}_{1} B$ креативно. Противоречие.

Пусть $\bar{R}_{1} \cap \bar{K}$ продуктивно. Тогда $A \cup R_{1}$ креативно и, следовательно, $A$ креативно. Вновь получили противоречие.

2) Пусть $|C \backslash H|=\infty$. Множество

$$
H \cap\left(\bigcup_{x \in \omega} \bigcup_{y \in D_{f(x)}} D_{y}\right)
$$


бесконечно. Поэтому существует одно-однозначная ОР $\Phi g$ такая, что

$$
g(\omega)=H \cap\left(\bigcup_{x \in \omega} \bigcup_{y \in D_{f(x)}} D_{y}\right) .
$$

Определим $\mathrm{OP} \Phi f_{1}$ следующим образом:

$D_{f_{1}(0)}=D_{f(0)}, \quad D_{f_{1}(n+1)}=\left\{y:(\exists z)\left(z \in D_{f(n+1)} \& D_{y}=D_{z} \backslash\{g(0), \ldots, g(n)\}\right)\right\}$

для любого $n \geqslant 0$. Тогда для каждого $m \in \omega$

$$
\left|\left\{x:(\exists y)\left(y \in D_{f_{1}(x)} \& D_{y} \cap\left(H \cap\left(\bigcup_{i \leqslant m} \bigcup_{z \in D_{f_{1}(i)}} D_{z}\right)\right) \neq \varnothing\right)\right\}\right|<\infty .
$$

Действительно, пусть $t$ - число такое, что

$$
H \cap\left(\bigcup_{i \leqslant m} \bigcup_{z \in D_{f_{1}(i)}} D_{z}\right) \subseteq\{g(0), \ldots, g(t)\}
$$

Тогда

$$
(\forall s)\left(s>t \Longrightarrow(\forall y)\left(y \in D_{f_{1}(s)} \Longrightarrow D_{y} \cap\left(H \cap\left(\bigcup_{i \leqslant m} \bigcup_{z \in D_{f_{1}(i)}} D_{z}\right)\right)=\varnothing\right)\right) .
$$

Очевидно, что

$$
\begin{gathered}
(\forall x)\left(x \in A \Longleftrightarrow(\exists y)\left(y \in D_{f_{1}(x)} \& D_{y} \subset H\right)\right), \\
\left|\left(\bigcup_{x \in B} \bigcup_{y \in D_{f_{1}(x)}} D_{y}\right) \backslash H\right|=\infty .
\end{gathered}
$$

Пусть $g_{1}$ - одно-однозначная ОР $\Phi$ и $g_{1}(\omega)=B$. Определим ОР $\Phi$ следуюшим образом. Положим по определению

$$
D_{h(0)}=\bigcup_{y \in D_{f_{1} g_{1}(0)}} D_{y}
$$

Пусть значения $h(0), h(1), \ldots, h(n)$ определены. Тогда берем наименьшее число $s$ такое, что

$$
(\forall y)\left(y \in D_{f_{1} g_{1}(s)} \Longrightarrow D_{y} \cap\left(\bigcup_{x \leqslant n} D_{h(x)}\right)=\varnothing\right)
$$


и полагаем

$$
D_{h(n+1)}=\bigcup_{y \in D_{f_{1} g_{1}(s)}} D_{y}
$$

Такое $s$ всегда найдется. Допустим, что для некоторого $n$ такое $s$ не сушествует, т.е. для каждого $s \in \omega$ имеем

$$
(\exists y)\left(y \in D_{f_{1} g_{1}(s)} \& D_{y} \cap\left(\bigcup_{x \leqslant n} D_{h(x)}\right) \neq \varnothing\right)
$$

Рассмотрим множество

$$
R_{2}=\left\{x:(\exists y)\left(y \in D_{f_{1}(x)} \& D_{y} \cap\left(\bigcup_{x \leqslant n} D_{h(x)}\right) \neq \varnothing\right)\right\}
$$

Множество $R_{2}$ рекурсивное, $B \subseteq R_{2}$ и $\left|R_{2} \cap A\right|<\infty$. Множество $R_{2} \cap \bar{K}$ продуктивно или множество $\bar{R}_{2} \cap \bar{K}$ продуктивно. Тогда, рассуждая как и вьше, приходим к противоречию.

\section{СПИСОК ЦИТИРОВАННОЙ ЛИТЕРАТУРЫ}

[1] Lerman M., Remmel J. B. The universal splitting property. I // Logic Colloqium '80 / ed. D. van Dalen et al. Stud. Logic Found. Math. V. 108. Amsterdam: Nort-Holland, 1982. P. 181-207.

[2] Lerman M., Remmel J. B. The universal splitting property. II // J. Symbolic Logic. 1984. V. 49. №1. P. 137-150.

[3] Downey R. G. The degree of r.e. sets without the universal splitting property // Trans. Amer. Math. Soc. 1985. V. 291. P. 337-351.

[4] Ambos-Spies K., Fejer P. A. Degree theoretical splitting properties of recursively enumerable sets // J. Symbolic Logic. 1988. V. 53. № 4. P. 110-137.

[5] Downey R. G., Stob M. Automorphisms of the lattice of recursively enumerable sets: Orbits // Adv. Math. 1992. V. 92. P. 237-265.

[6] Роджерс Х. Теория рекурсивных функций и эффективная вычислимость. М.: Мир, 1972.

[7] Soare R. I. Recursively Enumerable Sets and Degrees. Berlin-New York-Tokyo: Springer, 1987.

[8] Downey R. G., Jockusch C. G. T-degrees, jump classes and strong reducibilities // Trans. Amer. Math. Soc. 1987. V. 301. № 1. P. 103-136.

[9] Оманадзе Р.Ш. О верхней полурешетке рекурсивно перечислимых $s Q$-степеней // Алгебра и логика. 1991. Т. 30. № 4. С. 405-413.

[10] Jockusch C. G. Semirecursive sets and positive reducibility // Trans. Amer. Math. Soc. 1968. V. 131. № 2. P. 420-436.

[11] Марченков С. С. Об одном классе неполных множеств // Матем. заметки. 1976. Т. 20. № 4. C. 473-478.

[12] Оманадзе Р.Ш. Соотношения между некоторыми сводимостями // Алгебра и логика. 1994. Т. 34. №6. С. 681-688.

[13] Оманадзе Р. Ш. О верхней полурешетке рекурсивно перечислимых $Q$-степеней // Алгебра и логика. 1984. Т. 23. №2. С. 175-184. 
[14] Оманадзе Р. Ш. Об одном усилении $Q$-сводимости // Алгебра и логика. 1995. Т. 35. № 1. C. $79-87$.

[15] Shore A. R. Nowhere simple sets and the lattice of recursively enumerable sets // J. Symbolic Logic. 1978. V. 43. № 2. P. 322-330.

[16] Оманадзе Р. Ш. Q-сводимость и нигде не простые множества // Сообщ. АН ГССР. 1987. T. 127. №1. C. 29-32.

[17] Yates G. E. M. Three theorems of the degrees of recursively enumerable sets // Duke Math. J. 1965. V. 32. № 3. P. 461-468.

[18] Ditshev A. V. Some results on bounded truth-table degrees // Z. Math. Logik Grundlag. Math. 1990. V. 36. №3. P. 263-271.

[19] Blum M., Marques I. On complexity properties of recursively enumerable sets // J. Symbolic Logic. 1973. V. 38. № 4. P. 579-593.

[20] Lachlan A. H. A note on universal sets // J. Symbolic Logic. 1966. V. 31. № 3. P. 573-574.

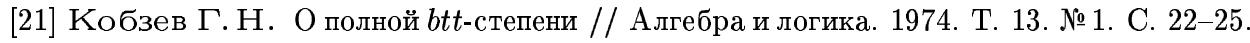

Тбилисский государственньй университет им. И. А. Джавахишвили

Поступило

Институт прикладной математики им. И. Н. Векуа

27.06 .95

E-mail : omanr@viam.hepi.edu.ge

Исправленньй вариант 12.09 .98 